\title{
Inflammatory myofibroblastic tumor as adverse outcome of eosinophilic esophagitis
}

An emergency esophagogastroduodenoscopy performed in a dyspeptic 19-yearold girl with hematemesis, abdominal pain, and anemia ( $\bullet$ Fig. 1) revealed a polypoid esophageal mass with distal erosion. A subsequent $\mathrm{CT}$ scan confirmed the bulky lesion $(14 \times 4 \times 3.5 \mathrm{~cm})$ extending from the carina to the cardia level. Both extraesophageal extension and nodal and extranodal metastases were excluded. The extent and location of the mass ruled out any endoscopic resection and a total esophagectomy with gastric pull-up was performed. Both histology and immunophenotyping (positive for vimentin, MNF116, and smooth muscle actin; negative for S100 and CD117) were consistent with the "classical variant" of inflammatory myofibroblastic tumor (IMT). A prominent eosinophilic infiltrate ( Fig. 2a-c) was also present, spreading from the lesional to the extralesional area, where the squamous epithelium featured rich eosinophic infiltrate, fully consistent with eosinophilic esophagitis (॰ Fig. 2d).

Within the upper gastrointestinal tract, IMTs are rare [1], polymorphic clinicopathological entities that have been associated with gastric ulcers [2], ischemic disease, and gastroesophageal reflux [3]. In accordance with these clinical settings, the hypothesis of IMT as an "exaggerated reparative response" has been established. In eosinophilic esophagitis, the promoting role for eosinophils in the onset of subepithelial fibrosis is supported by the high transcript levels of both interleukin-5 and TGF- $\beta$ detected in tissue samples from lesions [3-5]. Most recently, this hypothesis has been validated in murine models of eosinophilic esophagitis, where eosinophilia leads to collagen accumulation, finally resulting in esophageal wall remodeling [5]. A causative role for eosinophilic esophagitis is further sustained by the clinical finding that more than $50 \%$ of cases of eosinophilic esophagitis coexist with fibrotic esophageal strictures (e.g., Schatzki rings and esophageal webs), which might be seen as part of the same "tumorigenic field" in which IMT may develop.

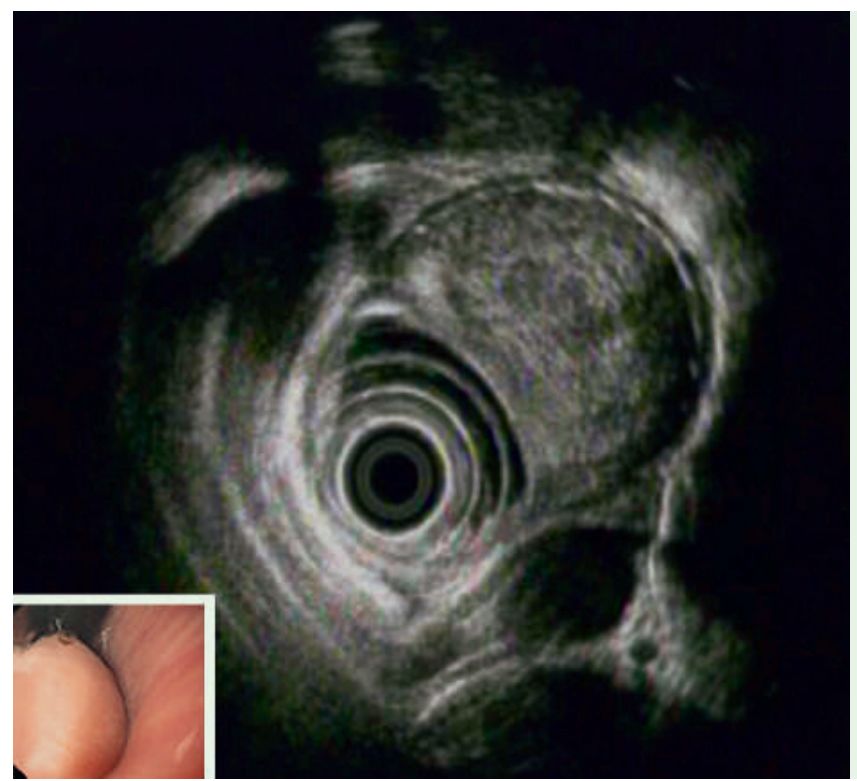

Fig. 1 Endoscopic ultrasonography showing a nonhomogeneous solid mass originating from the inner esophageal layers and involving mucosa and submucosa.
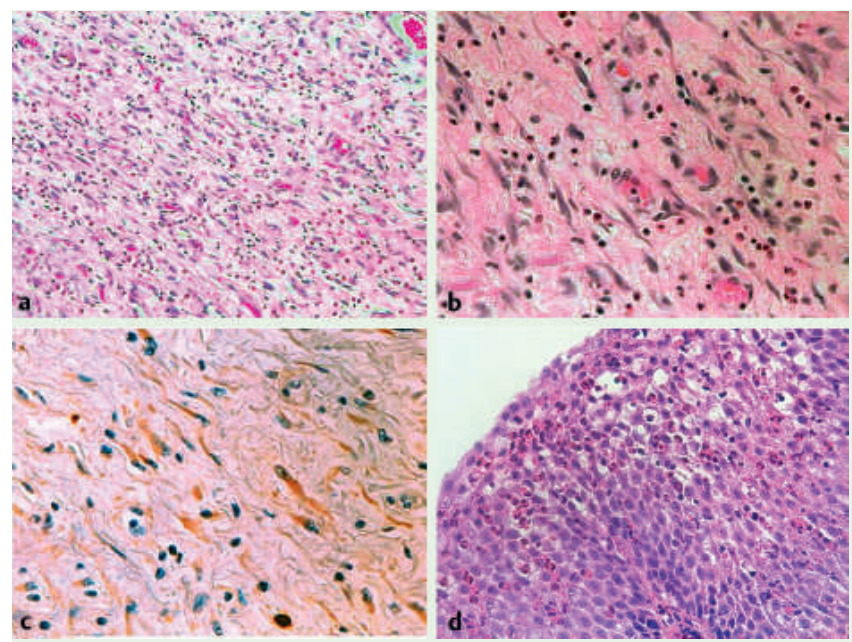

Fig. 2 a-d Histological and immunohistochemical features observed in the present case.

a Solid inflammatory myofibroblastic tumor (IMT) consisting of spindle cells included in a myxoid, richly vascularized stroma (hematoxylin \& eosin, original magnification $\times 10$ ). b Prominent eosinophilic intratumoral infiltrate (hematoxylin \& eosin, original magnification $\times 40$ ). c Neoplastic spindle cells exhibit positive immunoreaction to anti-smooth-muscle actin monoclonal antibody (original magnification $\times 40$ ). d Eosinophilic esophagitis (hematoxylin \& eosin, original magnification $\times 20$ ).

The clinical observation of a young patient with concomitant eosinophilic esophagitis and IMT, supported by recent experimental and molecular evidence, suggests that IMTs can definitely be considered among the unfavorable outcomes of (long-standing) eosinophilic esophagitis.

Endoscopy_UCTN_Code_CCL_1AB_2AC_3AB
M. Fassan ${ }^{1}$, C. Castoro ${ }^{2}$, A. J. Saenz ${ }^{4}$, M. Cagol ${ }^{3}$, V. Ninfo ${ }^{1}$, M. Rugge ${ }^{1}$

Pathology Unit, Department of Medical Diagnostic Sciences and Special Therapies, University of Padua, Italy Surgery Unit, Istituto Oncologico Veneto IOV-IRCCS, Padua, Italy Surgery Unit, Department of Medical and Surgical Sciences, University of Padua, Italy 
4 James Homer Wright Pathology Laboratories, Massachusetts General Hospital, Harvard Medical School, Boston,

Massachusetts, USA

\section{References}

1 Makhlouf HR, Sobin LH. Inflammatory myofibroblastic tumors (inflammatory pseudotumors) of the gastrointestinal tract: how closely related to inflammatory fibroid polyps? Hum Pathol 2002; 33: 307-315

2 Saccà N, Rodinò S, DeMedici A et al. NSAIDinduced digestive hemorrhage and esophageal pseudotumor: a case report. Endoscopy $1995 ; 27: 632$
3 Staples DC, Knodell RG, Johnson LF. Inflammatory pseudotumor of the esophagus. A complication of gastroesophageal reflux. Gastrointest Endosc 1978; 24: 175 - 176

4 Chehade M, Sampson HA, Morotti RA et al. Esophageal subepithelial fibrosis in children with eosinophilic esophagitis. J Pediatr Gastroenterol Nutr 2007; 45: 319-328

5 Mishra A, Wang M, Pemmaraju VR et al. Esophageal remodeling develops as a consequence of tissue specific IL-5-induced eosinophilia. Gastroenterology 2008; 134: 204-214
Bibliography

DOI 10.1055/s-2008-1077646

Endoscopy 2009; 41: E95 - E96

(c) Georg Thieme Verlag KG Stuttgart · New York . ISSN 0013-726X

\section{Corresponding author}

\section{Rugge, MD}

II Cattedra di Anatomia Patologica Università degli Studi di Padova Istituto Oncologico Veneto-IRCCS Via Aristide Gabelli, 61

35121 Padova

Italy

Fax: +39-049-8272277

massimo.rugge@unipd.it 